\title{
HIV Risk Profiles Among HIV-Positive, Methamphetamine-Using Men Who Have Sex with Both Men and Women
}

\author{
Nadine Nakamura $\cdot$ Shirley J. Semple $\cdot$ \\ Steffanie A. Strathdee $\cdot$ Thomas L. Patterson
}

Received: 19 May 2010 / Revised: 18 October 2010 / Accepted: 23 October 2010 / Published online: 4 January 2011

(C) The Author(s) 2010. This article is published with open access at Springerlink.com

\begin{abstract}
This study examined demographic characteristics, sexual risk behaviors, sexual beliefs, and substance use patterns in HIV-positive, methamphetamine-using men who have sex with both men and women (MSMW) $(n=50)$ as compared to men who have sex with men only (MSM) $(n=150)$. Separate logistic regressions were conducted to predict group membership. In the final model, of 12 variables, eight were independently associated with group membership. Factors independently associated with MSMW were acquiring HIV through injection drug use, being an injection drug user, using hallucinogens, using crack, being less likely to have sex at a bathhouse, being less likely to be the receptive partner when high on methamphetamine, having greater intentions to use condoms for oral sex, and having more negative attitudes about HIV disclosure. These results suggest that, among HIV-positive methamphetamine users, MSMW differ significantly from MSM in terms of their HIV risk behaviors. Studies of gay men and HIV often also include bisexual men, grouping them all together as MSM, which may obscure important differences between MSMW and MSM. It is important that future studies consider MSM and MSMW separately in order to expand our knowledge about differential HIV prevention needs for both groups. This study
\end{abstract}

N. Nakamura

Faculty of Health Sciences, Simon Fraser University,

Vancouver, BC, Canada

S. J. Semple · T. L. Patterson $(\square)$

Department of Psychiatry (0680), University of California,

9500 Gilman Drive, La Jolla, CA 92093-0680, USA

e-mail: tpatterson@ucsd.edu

S. A. Strathdee

Division of Global Public Health, Department of Medicine,

University of California, La Jolla, San Diego, CA, USA showed that there were important differences in primary and secondary prevention needs of MSM and MSMW. These findings have implications for both primary and secondary HIV prevention among these high-risk populations.

Keywords Men who have sex with men and women . Men who have sex with men · Bisexual · HIV .

Methamphetamine $\cdot$ Injection drug use

\section{Introduction}

There has been much media hype and sensationalizing of men who have sex with men and women (MSMW) in the past decade, perhaps most famously with an episode of the Oprah Winfrey Show about men on the "down low" (Sandfort \& Dodge, 2008). Bisexuality was presented as a "shameful 'secret' which put innocent people (women in particular) at risk for disease and death" (Sandfort \& Dodge, 2008, p. 676). In the scientific literature, MSMW have been framed as a potential "bridge" that can place heterosexual women at risk for HIV infection (Deiss et al., 2008; Siegel, Schrimshaw, Lekas, \& Parsons, 2008). Some evidence does point to increased risk among MSMW, such as Prabhu, Owen, Folger, and McFarland's (2004) study, which found that unprotected anal intercourse between serodiscordant partners significantly decreased from 1998 to 2003 among MSM, but remained stable among MSMW. However, difference in serostatus and partner type has been found to be associated with differential risk behavior. For example, HIVpositive African American MSMW were less likely to have unprotected sex with male main partners and female main partners compared to HIV-negative or HIV-unknown MSMW, though they were just as likely to have unprotected sex with nonmain male and non-main female partners as HIV-negative or HIV-unknown MSMW (Lauby et al., 2008). These findings 
suggest that risk among MSMW is likely more nuanced than it has been portrayed and requires additional research.

An important issue to consider is that sexual identity and labeling differ across racial and ethnic groups. Ethnic and racial minority MSM, for example, are less likely to identify as gay compared to white men (Millet, Malebranche, Mason, \& Spikes, 2005; Pathela et al., 2006). Pathela et al. found that heterosexually-identified MSM who had sex exclusively with men were more likely than gay-identified MSM to be racial or ethnic minorities. These heterosexually-identified MSM were less likely to have been recently tested for HIV and were less likely to report condom use during their last sexual encounter when compared to gay-identified MSM. However, Millet et al. (2005) reported that while African American MSM were less likely to disclose that they have sex with men compared to other MSM, they engaged in less risky sexual behavior than MSM who did disclose their homosexual behavior. Thus, ethnic minority status and disclosure of sexual orientation alone may not be reliable barometers of risk.

Despite the hype about MSMW being especially risky, most studies of HIV risk lump MSM and MSMW together (Dodge \& Sandfort, 2007). Of those studies that have specifically examined differences between MSM and MSMW in relation to HIV, several risk factors have been identified that relate to sexual behavior and substance use (Dodge \& Sandfort, 2007). Studies indicate that MSMW are more likely than MSM to be injection drug users (IDUs) (Dodge, Jeffries, \& Sandfort, 2008; Goodenow, Netherland, \& Szalacha, 2002; Jeffries \& Dodge, 2007), to report trading sex for money or drugs (Jeffries \& Dodge, 2007), to have sex under the influence of drugs (Jeffries \& Dodge, 2007), and to have lower intentions to use condoms, weaker peer norms favoring safer sex, and more risk avoidance (Heckman et al., 1995).

Several recent studies have specifically compared HIVpositive MSMW and MSM (Hightow, Leone, MacDonald, McCoy, \& Sampson, 2006; Knight et al., 2007; Lauby et al., 2008; Montgomery, Mokotoff, Gentry, \& Blair, 2003; Mutchler et al., 2008; O'Leary, Purcell, Remien, Fisher, \& Spikes, 2007). These studies found that HIV-positive MSMW and MSM were different on some key variables, but not on others. For example, HIV-positive MSMW were more likely to be sexually compulsive (O'Leary et al., 2007), tended to be less involved with the gay community (O'Leary et al., 2007), and were younger and more likely to be African American compared to HIV-positive MSM (Hightow et al., 2006; O'Leary et al., 2007). However, they did not differ on depression and anxiety (O'Leary et al., 2007) and they did not seem to seek out sex partners from different venues than MSM (O'Leary et al., 2007).

Methamphetamine has been implicated as being highly associated with risky sexual behavior among MSM in numerous studies (Colfax \& Shoptaw, 2005; Drumright et al., 2006; Fernández et al., 2007; Plankey et al., 2007; Purcell, Moss, Remien, Woods, \& Parsons, 2005; Vaudrey et al., 2007). For example, Drumright et al. reported a five-fold increase in unprotected sex in those HIV-positive MSM who had used methamphetamine. However, no studies have examined methamphetamine-using MSMW specifically. Further, there have been no studies that examined differences between HIV-positive methamphetamine-using MSMW and MSM. Understanding possible differences between these high-risk populations may aid in the development of more effective interventions.

Recognizing that methamphetamine users are at especially high risk for HIV transmission, the purpose of the present study was to explore differences in risk factors between HIV-positive methamphetamine using MSM and MSMW. We examined several categories of risk factors identified by previous studies on HIV risk among MSMW, including demographic characteristics, substance use patterns (e.g., IDU), and sexual risk related factors (e.g., behaviors, disclosure) in a sample of HIVpositive MSMW and MSM methamphetamine users in San Diego, California.

\section{Method}

Participants

Data were collected between November 2000 and October 2004 during baseline assessments of men enrolled in a behavioral intervention study, as previously described (Patterson, Semple, Zians, \& Strathdee, 2005). This intervention consisted of eight individual counseling sessions to address risky sexual behavior of HIV-positive, methamphetamine-using MSM. Eligible participants were those who were confirmed to be HIV-positive, at least 18 years old, had unprotected anal or oral sex with at least one HIV-negative or serostatus-unknown male partner in the last 30 days, and used methamphetamine at least twice in the past 2 months and at least once in the last 30 days. Participants were recruited from the community through poster and media campaigns and street outreach.

The initial sample consisted of 250 gay-identified men who reported only having sex with a male partner and 50 men who reported having had both female and male sex partners in the past 2 months, of whom $72 \%$ self-identified as bisexual, $22 \%$ self-identified as gay, and $6 \%$ did not give a response. Of the 250 MSM, 150 were randomly selected as the comparison group in order to have a 3 to 1 comparison group. There was no significant difference between the included MSM and the excluded MSM in age, employment, sexual orientation, contracting HIV through unprotected sex, or contracting HIV through IDU.

\section{Procedure}

Participants were interviewed on various topics, including background characteristics, substance use, and sexual risk behaviors using face-to-face interviews. Participants received payment of 
$\$ 30$ for completing the baseline assessment and first counseling session.

\section{Measures}

\section{Classification by Gender of Sexual Partners}

By definition, due to the study's eligibility criteria, all participants indicated that they had sex with a man in the past 2 months. Participants were also asked if they had sex with a woman in the past 2 months. Those who indicated that they had sex with both men and women in the past 2 months were categorized as MSMW. Those who indicated that they had sex with only men were categorized as MSM.

\section{Demographic Characteristics}

Participants indicated their age, ethnicity, annual income, educational level, and the year they received their HIV diagnosis, and how they believed they contracted HIV. Education was measured as a categorical variable with the following categories: some high school or less, but no diploma, certificate, or GED; high school diploma or GED; 2-year degree, some college, or other non-military technical school training; 4-year degree; some graduate work or advanced degree.

\section{Psychosocial Factors}

Depressive symptoms were measured by the 21-item Beck Depression Inventory (BDI-II) (Beck, Steer \& Brown, 1996). The BDI consists of 21 items, each with four graded statements pertaining to how the participant has been feeling during the past week. The statements within a question were ordered 0 to 3 to show increasing depressive symptoms. Summary scores range from 0 to 63. Cronbach's alpha for the BDI in this sample was 90. Sexual compulsivity was measured using the 10-item selfreport compulsivity scale developed by Kalichman et al. (1994). Items in this scale reflect the extent to which participants agree with statements about sexually compulsive behavior, sexual preoccupations, and sexually intrusive thoughts (Kalichman et al., 1994; Kalichman \& Rompa, 2001). Responses to items on this scale ranged from $1=$ "Not at all like me" to $4=$ "Very much like me." Alpha reliability for this scale with our sample was .91.

\section{Substance Use}

Participants were asked about their methods of methamphetamine use, how many days in the past 30 they used methamphetamine, the total quantity they used in the past month, and how many times they used in a typical day. They were also asked whether they had used other substances in the past 2 months, including alcohol, marijuana, cocaine, crack, amyl nitrates, hallucinogens, heroin, and GHB.

\section{Sexual Behavior}

Participants were asked how many times they had had anal and oral sex in the past 2 months with and without condoms. They were asked to indicate whether they were more likely to be the receptive partner, to be sexually disinhibited, to have vigorous sex to the point of the condom breaking, and to have anal and oral sex without condoms when high on methamphetamine. Participants were also asked about different locations they frequented to have sex, including bathhouses and parks.

\section{Intentions to Use Condoms}

Participants' intentions to use condoms were assessed with two questions: "I intend to always use condoms during anal intercourse during the next 2 months" and "I intend to always use a condom or dental dam during oral sex during the next 2 months." Responses to these items ranged from $1=$ "Very untrue" to $5=$ "Very true" (Fisher, Willcuts, Misovich, \& Weinstein, 1998).

\section{Negative Attitudes Toward Condom Use}

Participants' negative attitudes on this topic were calculated by summing responses to the following questions: (1) "I believe that using condoms interferes with sexual pleasure"; (2) "I believe that stopping to put on a condom ruins the moment"; (3) "I believe that using condoms makes me less sexually desirable"; (4) "Using condoms during sex ruins the mood"; (5) "Using a condom will feel unnatural"; and (6) "My partner(s) will not be sexually satisfied if we use a condom" (Mausbach, Semple, Strathdee, \& Patterson, 2009). Responses to items on this scale ranged from $1=$ "Strongly Disagree" to $4=$ "Strongly Agree." Alpha reliability for this scale with our sample was .85.

\section{Attitudes About HIV Disclosure}

Participants' attitudes about disclosing their HIV serostatus were measured using an 11-item self-report scale. Sample items included, "I believe that disclosing my HIV status to my sexual partner(s) will increase my sexual pleasure" and "I believe that my sexual partner(s) will not trust me if I tell him/her that I am HIV positive." Responses to items on this scale ranged from $1=$ "Strongly Disagree" to $4=$ "Strongly Agree." Alpha reliability for this scale with our sample was .85 .

\section{Data Analysis}

Data were examined for normality of distribution, and log 10 transformations were performed when violations were detected. Continuous data were analyzed using independent sample $t$-tests. Categorical data were analyzed with Pearson chi-square tests. All statistical tests were two-tailed. 
Separate logistic regressions were run to predict group membership ( $1=$ MSMW, $0=$ MSM). Demographic characteristics that were significant $(p<.05)$ at the univariate level were entered into the first logistic regression equation. In the second equation, drug variables that were significant $(p<.05)$ at the univariate level were entered into the second logistic regression. In the third equation, sexual risk variables that were significant $(p<.05)$ at the univariate level were entered into a third logistic regression. In a final equation, all variables that were significant $(p<.05)$ in the first three regressions were entered, including ethnicity.

\section{Results}

Demographic Characteristics

Of the 200 male participants, mean age was 37 years $(S D=$ 7.42). The majority of participants were Caucasian $(53.5 \%)$, 23.5\% were African American, $13.5 \%$ were Latino, and $9.5 \%$ were "other" races. MSMW were more likely to be African American and to have less than a high school diploma compared to MSM (Table 1), but they did not significantly differ from MSM on age or income. MSM were more likely to report contracting HIV from sexual contact, while MSMW were more likely to report contracting HIV through drug use. MSM tended to be diagnosed with HIV at a younger age than MSMW. The effect sizes for being African American, having less than a high school diploma, acquiring HIV through sexual contact, and depressive symptoms were small, while acquiring HIV through drug use had a moderate effect size.

\section{Substance Use}

As shown in Table 1, in the past 30 days, MSMW used methamphetamine for a greater number of days and used more grams of methamphetamine compared to MSM. MSMW were also more likely to use methamphetamine intravenously. MSMW were more likely to use alcohol, marijuana, cocaine, crack, hallucinogens, and heroin before or during sex compared to MSM, whereas the latter group was more likely to use amyl nitrates and GHB. The effect sizes of these variables were small, with the exception of number of days of meth use in the past 30 days, injection methamphetamine use, and cocaine and crack use in the past 2 months, which were moderate.

\section{Sexual Risk}

MSMW and MSM did not differ significantly in their number of reported unprotected anal or oral sex acts. MSM reported that they were more likely to be the receptive partner, to be sexually disinhibited, and to have anal and oral sex without condoms when high on methamphetamine compared to MSMW. MSMW scored significantly higher on having vigorous sex where the condom breaks when they were high on methamphetamine compared to MSM. MSM were more likely to have sex at bathhouses compared to MSMW, while MSMW were more likely to have sex in parks compared to MSM. MSMW and MSM did not significantly differ in sexual compulsivity scores or in their attitudes about condoms. However, MSMW scored significantly higher on intentions to use condoms for both oral and anal sex compared to MSM. Finally, MSMW had significantly more negative attitudes about HIV disclosure to sexual partners compared to MSM. The effect sizes of these variables were small, with the exception of being the receptive partner when high on meth and intentions to use condoms for oral sex, which were moderate.

\section{Correlates of MSMW Group Membership}

In the first equation, six background characteristics that were significant on the univariate level were entered into a logistic regression (Table 2). These variables were ethnicity (White $=0$; Other $=1$ ), education, HIV contracted through sex, acquiring HIV through injection drug use, age at HIV diagnosis, and depressive symptoms. Contracting HIV through injecting drugs predicted MSMW group membership.

In the second equation, 12 drug use variables that were significant in univariate analyses were entered into a logistic regression model. These variables were injection meth use, amount of meth used in the past 30 days, number of times meth was used in a typical day, number of days of meth use in the past 30 days, alcohol use, marijuana use, cocaine use, crack use, amyl nitrate use, hallucinogen use, heroin use, and GHB use. Injecting meth, using alcohol, using crack, using hallucinogens, and not using amyl nitrates significantly predicted MSMW group membership.

In the third equation, 10 sexual risk variables that were significant in univariate analyses were entered into a logistic regression model. These variables were having anal sex without a condom when high on meth, being the receptive partner when high on meth, being sexually disinhibited when high on meth, not using a condom for oral sex when high on meth, having vigorous sex where the condom breaks when high on meth, having sex in a bathhouse, having sex in a park, intentions to use condoms for oral sex, intentions to use condoms for anal sex, and negative attitudes about HIV disclosure. Having vigorous sex to the point of the condom breaking when high on meth, not having sex at a bathhouse, higher intentions to use condoms for oral sex, and having more negative attitudes about HIV disclosure significantly predicted being in the MSMW group. Being more likely to be the receptive partner when high on meth predicted being in the MSM group.

In the final equation, all 11 variables that were significant in the first three regressions were entered. Ethnicity was also included in the final equation since the literature suggests that ethnic minority MSM often differ from Caucasian MSM on sexual risk outcomes 
Table 1 Characteristics of HIV-positive MSMW and MSM methamphetamine users

\begin{tabular}{|c|c|c|c|c|c|c|c|c|c|}
\hline Variable & $\begin{array}{l}\text { MSMW } \\
(\% / M)\end{array}$ & $n$ & $S D$ & $\begin{array}{l}\text { MSM } \\
(\% / M)\end{array}$ & $n$ & $S D$ & Test statistic & $\begin{array}{l}\text { Effect } \\
\text { size }^{\mathrm{a}}\end{array}$ & \\
\hline African American & $34.0 \%$ & 17 & - & $20.0 \%$ & 30 & - & $\chi^{2}=4.09 *$ & .14 & \\
\hline Some high school education or less & $26.0 \%$ & 13 & - & $13.3 \%$ & 20 & - & $\chi^{2}=4.37 *$ & .15 & \\
\hline Acquired HIV through sexual contact & $70.0 \%$ & 35 & - & $92.7 \%$ & 139 & - & $\chi^{2}=17.04 * * * *$ & -.29 & \\
\hline Acquired HIV through drug use & $48.0 \%$ & 24 & - & $15.3 \%$ & 23 & - & $\chi^{2}=22.26 * * * *$ & .33 & \\
\hline Injection meth use & $72.0 \%$ & 36 & - & $33.3 \%$ & 50 & - & $\chi^{2}=22.88 * * * *$ & .34 & \\
\hline Alcohol use in past 2 months & $92.0 \%$ & 46 & - & $76.7 \%$ & 115 & - & $\chi^{2}=5.62 *$ & .17 & \\
\hline Marijuana use in past 2 months & $86.0 \%$ & 43 & - & $64.7 \%$ & 97 & - & $\chi^{2}=8.13^{*}$ & .20 & \\
\hline Cocaine use in past 2 months & $60.0 \%$ & 30 & - & $24.7 \%$ & 37 & - & $\chi^{2}=21.02 * * * *$ & .32 & \\
\hline Crack use in past 2 months & $50.0 \%$ & 25 & - & $14.7 \%$ & 22 & - & $\chi^{2}=26.04 * * * *$ & .36 & \\
\hline Amyl nitrate use in the past 2 months & $46.0 \%$ & 23 & - & $64.7 \%$ & 97 & - & $\chi^{2}=5.44 *$ & .17 & \\
\hline Hallucinogen use in the past 2 months & $24.0 \%$ & 12 & - & $6.7 \%$ & 10 & - & $\chi^{2}=11.51^{*}$ & .24 & \\
\hline Heroin use in the past 2 months & $20.0 \%$ & 10 & - & $4.0 \%$ & 6 & - & $\chi^{2}=13.04 * * *$ & .26 & \\
\hline GHB use in the past 2 months & $14.0 \%$ & 7 & - & $29.3 \%$ & 44 & - & $\chi^{2}=4.64 *$ & -.15 & \\
\hline Sex in bathhouse & $18 \%$ & 9 & - & $46 \%$ & 69 & - & $\chi^{2}=12.36 * * * *$ & -.25 & \\
\hline Sex in park & $50 \%$ & 25 & - & $27 \%$ & 41 & - & $\chi^{2}=8.71 * *$ & .21 & \\
\hline Age at HIV diagnosis ${ }^{\mathrm{b}}$ & 32.3 & 47 & 8.76 & 29.6 & 150 & 7.40 & $t=2.07 *$ & .02 & 19 \\
\hline Number of depressive symptoms ${ }^{c}$ & 18.26 & 46 & 9.41 & 14.92 & 143 & 10.32 & $t=1.95^{*}$ & .17 & 18 \\
\hline Amount of meth used in the past 30 days (in grams) ${ }^{\mathrm{d}}$ & 9.27 & 49 & 17.25 & 4.54 & 148 & 10.38 & $t=-1.81^{*}$ & .16 & 19 \\
\hline Number of days of meth use in the past 30 days $^{\mathrm{e}}$ & 14.86 & 50 & 10.06 & 9.08 & 148 & 8.33 & $t=3.66 * * * *$ & .30 & 19 \\
\hline Number of times meth used in a typical day ${ }^{\mathrm{f}, \mathrm{g}}$ & 5.10 & 49 & 5.10 & 5.00 & 139 & 4.08 & $t=-0.14$ & .01 & 18 \\
\hline Have more anal sex without condoms when high on meth & 2.32 & 50 & 1.11 & 2.74 & 150 & 1.13 & $t=-2.29^{*}$ & -.18 & 19 \\
\hline Receptive partner when high on meth ${ }^{\mathrm{h}}$ & 2.33 & 50 & 1.18 & 3.09 & 150 & .941 & $t=-4.60 * * * *$ & -.34 & 19 \\
\hline Sexually disinhibited when high on meth ${ }^{\mathrm{h}}$ & 3.12 & 49 & 1.01 & 3.48 & 148 & .892 & $t=-2.35^{*}$ & -.19 & 195 \\
\hline Use a condom for oral sex when high on meth ${ }^{\mathrm{h}}$ & 2.90 & 49 & 1.10 & 3.30 & 150 & 1.05 & $t=-2.30^{*}$ & -.18 & 197 \\
\hline Vigorous sex where condom breaks when high on meth ${ }^{\mathrm{h}}$ & 2.52 & 50 & 1.02 & 2.15 & 149 & 1.07 & $t=2.15^{*}$ & -.17 & 197 \\
\hline Intentions to use condoms for oral $\operatorname{sex}^{\mathrm{i}}$ & 3.30 & 50 & 1.57 & 1.96 & 149 & 1.25 & $t=6.13 * * * *$ & .43 & 197 \\
\hline Intentions to use condoms for anal sex ${ }^{\mathrm{i}}$ & 3.70 & 50 & 1.49 & 3.16 & 149 & 1.49 & $t=2.22^{*}$ & .18 & 197 \\
\hline Negative attitudes about HIV disclosure ${ }^{\mathrm{j}}$ & 29.52 & 50 & 7.57 & 25.84 & 149 & 7.31 & $t=3.06^{*}$ & .24 & 197 \\
\hline Total number of partners in the past 2 months ${ }^{\mathrm{k}}$ & 9.73 & 45 & 11.30 & 11.30 & 137 & 17.93 & $t=-8.09$ & -.06 & 18 \\
\hline
\end{tabular}

$* p<.05 ; * * p<.01 ; * * * p<.001 ; * * * * p<.0001$

${ }^{\text {a }}$ Effect size was calculated for continuous variables with effect-size $r$ and for dichotomous variables with phi

b Absolute range, 10-53

c Absolute range, 0-63

d Absolute range, 0-60

e Absolute range, 1-30

f This variable was not normally distributed and was significant at the .05 level as a transformed variable. The means for this variable are reported for the untransformed variable to enhance interpretability

g $p>.05$

$\mathrm{h}$ Absolute range, 1-4

I Absolute range, 1-5

j Absolute range, 1-4

k Absolute range, 1-167

and how they identify their sexual orientation (Millet et al., 2005; Muñoz-Laboy \& Dodge, 2007; Pathela et al., 2006). Having acquired HIV through injection drug use, being an injection drug user, using hallucinogens, using crack, not having sex at a bathhouse, being less likely to be the receptive partner when high on methamphetamine, higher intentions to use condoms for oral sex, and having more negative attitudes about HIV disclosure significantly predicted being in the MSMW group (Table 3). 
Table 2 Summaries of separate logistic regression analyses associated with MSMW group membership: Background characteristics $(n=179)$ (Eq. 1), drug use variables $(n=195)$ (Eq. 2), and sexual risk variables $(n=192)$ (Eq. 3)

$$
\begin{aligned}
& { }^{*} p<.05 ; * * p<.01 ; * * * p<.001 \\
& \text { a }{ }^{\text {a }} \text { White }=0 ; \text { Other }=1 \\
& \text { b } B=-1.20 ; \chi^{2}=35.03 ; d f=6 ; \\
& R^{2}=.17 \\
& { }^{\mathrm{c}} B=-1.09 ; \chi^{2}=89.56 ; \\
& d f=12 ; R^{2}=.37 \\
& { }^{\mathrm{d}} B=-1.10 ; \chi^{2}=74.52 ; \\
& d f=10 ; R^{2}=.32
\end{aligned}
$$

\begin{tabular}{|c|c|c|}
\hline Final model & $\begin{array}{l}\text { Adjusted odds } \\
\text { ratio/exp B }\end{array}$ & $\begin{array}{l}95 \% \text { Confidence } \\
\text { interval }\end{array}$ \\
\hline \multicolumn{3}{|l|}{ Equation $1^{\mathrm{b}}$} \\
\hline Ethnicity $^{\mathrm{a}}$ & .79 & $.36-1.76$ \\
\hline Education & .76 & $.54-1.09$ \\
\hline HIV acquired through sexual contact & .54 & $.13-2.23$ \\
\hline HIV acquired through drug use & $5.14 *$ & $1.16-22.78$ \\
\hline Age of HIV diagnosis (per year increase) & 1.05 & $1.00-1.11$ \\
\hline Mean number of depressive symptoms & 1.03 & $.99-1.07$ \\
\hline \multicolumn{3}{|l|}{ Equation $2^{\mathrm{c}}$} \\
\hline Injection meth use & $5.21 * *$ & $1.86-14.57$ \\
\hline Amount of meth used in past 30 days & .36 & $.09-1.51$ \\
\hline Number of times meth used in a typical day & 2.29 & $.19-26.91$ \\
\hline Number of days of meth use in the past 30 days & 1.07 & $.95-1.20$ \\
\hline Alcohol use & $5.74 *$ & $1.24-26.62$ \\
\hline Marijuana use & 2.47 & $.83-7.30$ \\
\hline Cocaine use & 2.54 & $.82-7.86$ \\
\hline Crack use & $3.25^{*}$ & $1.04-1.11$ \\
\hline Amyl nitrate use & $.26 * *$ & $.10-.70$ \\
\hline Hallucinogen use & $6.61 * *$ & $1.64-26.61$ \\
\hline Heroin use & 1.47 & $.36-5.97$ \\
\hline GHB use & .35 & $.09-1.31$ \\
\hline \multicolumn{3}{|l|}{ Equation $3^{\mathrm{d}}$} \\
\hline More anal sex without condoms when high on meth & 1.00 & $.61-1.64$ \\
\hline Receptive partner when high on meth & $.54 * *$ & $.36-.81$ \\
\hline Sexually disinhibited when high on meth & .74 & $.46-1.19$ \\
\hline Use a condom for oral sex when high on meth & .73 & $.45-1.17$ \\
\hline Vigorous sex (condom breaks) when high on meth & $1.71 *$ & $1.05-2.76$ \\
\hline Sex at a bathhouse & $.29 *$ & $.11-.79$ \\
\hline Sex at a park & 2.07 & $.85-5.01$ \\
\hline Intentions to use condoms for oral sex & $1.99 * * *$ & $1.37-2.90$ \\
\hline Intentions to use condoms for anal sex & .85 & $.56-1.28$ \\
\hline Negative attitudes about HIV disclosure & $1.07 *$ & $1.01-1.13$ \\
\hline
\end{tabular}

\section{Discussion}

Our study of HIV-positive methamphetamine using men found that MSMW differed from MSM in several ways, suggesting that these men have different HIV and STI prevention needs. Compared to MSM, MSMW were more likely to report a number of higher risk behaviors (such as injecting drugs and using a wider variety of illicit substances) that have implications for primary prevention of STI acquisition. MSM were also more likely to report being the receptive partner when high on methamphetamine, having poorer intentions to use condoms for oral sex, and having sex at bathhouses, while MSMW had significantly more negative attitudes about HIV disclosure. These findings have implications for secondary HIV prevention.

Our data suggest that HIV-positive MSM and MSMW face different obstacles to protecting their sexual partners. HIVpositive MSMW in our sample were less likely than MSM to take the receptive role for anal sex when high on methamphetamine and had more negative attitudes about disclosing their seropositive status, which would appear to heighten their partner's risk of acquiring HIV through unprotected anal sex. This may partially be explained by an earlier finding that non-gay identifying MSM often avoid taking the receptive sexual role with male partners, reflecting the belief that only the receptive partner is gay (Finlinson, Colon, Robles, \& Soto, 2006). Future research should examine attitudes about sexual roles as they pertain to MSMW. An HIV-positive man in the insertive role is more at risk of transmitting HIV or STIs to his partner than if he were to take the receptive role (Hart, Wolitski, Purcell, Gomez, \& Halkitis, 2003). MSMW's higher intentions to use condoms for oral sex might reflect their desire to avoid disclosure of their HIV status; planning to use condoms may help MSMW to feel less guilty about not disclosing their HIV status. Since MSMW have higher intentions to use condoms for oral sex compared to 
Table 3 Summary of binomial stepwise logistic regression analysis predicting group membership (MSMW) $(n=187)$

\begin{tabular}{lcc}
\hline Final model $^{\text {a }}$ & $\begin{array}{c}\text { Odds } \\
\text { ratio }\end{array}$ & $\begin{array}{l}95 \% \text { Confidence } \\
\text { interval }\end{array}$ \\
\hline Set 1: & .47 & $.16-1.38$ \\
Ethnicity & $4.77 *$ & $1.29-17.63$ \\
HIV through drug use & & \\
Set 2: & $3.28^{*}$ & $1.09-9.90$ \\
Injection meth use & 4.24 & $.88-20.55$ \\
Alcohol use & $3.41^{*}$ & $1.03-11.31$ \\
Crack use & .56 & $.20-1.57$ \\
Amyl nitrate use & $6.38^{*}$ & $1.38-29.60$ \\
Hallucinogen use & & \\
Set 3: & $.59 *$ & $.37-.94$ \\
Receptive partner when high on meth & 1.54 & $.91-2.59$ \\
Vigorous sex where condom breaks & & \\
$\quad$ when high on meth & $.28 *$ & $.09-.85$ \\
Sex at a bathhouse & $1.94 * * *$ & $1.33-2.82$ \\
Intentions to use condoms for oral sex & $1.08^{*}$ & $1.01-1.16$ \\
Negative attitudes about HIV disclosure & & \\
\hline$* p<.05 ; * * *<.001$ & & \\
a $B=-1.11 ; \chi^{2}=209.38 ; d f=12 ; R^{2}=.43$ &
\end{tabular}

MSM, there appears to already be some receptiveness to condom use among MSMW. It may be useful to address the risk of unprotected anal sex to receptive partners who are unaware of their HIV status as a part of HIV-prevention interventions for HIV-positive MSMW. Motivational interviewing can be an important tool to address the cognitive dissonance that may be experienced by members of this group who do not wish to disclose their HIV status, but wish to have insertive anal sex.

Even though the MSMW and MSM in our sample were all methamphetamine users, MSMW were more likely to report use of crack and hallucinogens. Studies of HIV-positive male crack users indicate that many trade sex for money or drugs with other men and condom use is low (Pallonen, Timpson, Williams, \& Ross, 2009; Timpson, Williams, Bowen, Atkinson, \& Ross, 2010). In a recent study by Baum et al. (2009), crack users were twice as likely to present a decline of CD4 cells independent of antiretroviral use, and viral loads were significantly higher for crack users independent of highly active antiretroviral therapy over time. Hallucinogens, such as GHB and ketamine, induce feelings of euphoria and are often used at circuit parties, being referred to as "club drugs" (Gorman \& Carroll, 2000; Romanelli, Smith, \& Pomeroy, 2003). Hallucinogen use among HIV-positive persons is particularly concerning, since it can compromise medication adherence, result in life-threatening drug interactions with antiretrovirals, and even compromise the immune system (Romanelli et al., 2003). Interventions targeting HIV-positive MSMW should help them understand the potential for various recreational drugs to compromise their health further given their HIV-status. In addition, crack users who are having male-to-male sex to support their drug addiction may not identify as gay or bisexual and may be better reached through interventions targeting substance users that address male sex work rather than through interventions for gay and bisexual men.

MSMW were more likely to report acquiring HIV through drug use and to be current injection drug users. This is consistent with previous reports of high rates of IDU by MSMW (for review, see Dodge \& Sandfort, 2007). MSMW who are IDUs may face discrimination for their drug use from the LGBT community as well as from the IDU community related to their sexual behavior. The MSMW in our sample were also living with the stigma of being HIV-positive. Previous research has demonstrated links between experiences of discrimination and negative mental and physical health outcomes among illicit drug users (Young, Stuber, Ahern, \& Galea, 2005). Continued injection drug use may reflect a negative coping response to the multiple stigmas of injection drug use, being HIV-positive, and being a MSMW. Outreach and prevention efforts aimed at IDUs may overlook MSMW and not address their specific needs. Interventions aimed at HIV-positive IDU MSMW should address the stressors of being members of stigmatized, marginalized groups. In addition, HIV prevention targeting methamphetamine-using MSMW should focus not only on safer sex behaviors, but also on safer injection practices.

MSMW were less likely to report having sex at a bathhouse than MSM. These findings suggest that methamphetamine-using, HIV-positive MSMW and MSM engage in risky behavior in different contexts, including different venues, which is contrary to an earlier study by O'Leary et al. (2007). HIV prevention campaigns that take place at bathhouses are not likely to reach MSMW, and research recruitment from these sites may unintentionally exclude MSMW.

A major limitation to our study was the way that MSMW were classified. We categorized men as MSMW based on whether they had sex with a woman in the past 2 months. It is possible that men classified as MSM had sex with women outside of that time frame. The sample of MSMW was relatively small, and a larger sample may have yielded more significant findings. A broader time frame may have yielded more participants in the MSMW category. The present study sample was not specifically recruited to examine differences between MSM and MSMW. The sample inclusion criterion was that participants had sex with men and some indicated that they also had sex with women. Future studies should specifically aim to recruit MSMW with the same timeframe criteria for sex with men and sex with women. An interesting finding in our data was that $22 \%$ of men who had sex with women in the past 2 months selfidentified as gay. This indicates that self-identity and sexual behavior even among gay men does not necessarily align. Questions about identity and behavior should both be included in interventions targeting gay men, leaving room for the possibility that just because one self-identifies as a gay man does not mean 
that he is not having sex with women. Given that less than $2 \%$ of the participants in the present study refused to indicate their sexual orientation and the remainder self-identified as gay or bisexual, our findings should not be generalized to include MSMW who identify as heterosexual.

This study showed that there were important differences in primary and secondary prevention needs of MSM and MSMW. Many previous studies of gay men included bisexual men, grouping all participants as MSM. While this might indicate a desire to include the experiences of MSMW, important differences between MSMW and MSM are obscured when they are combined for statistical purposes rather than examined separately. It is important that future studies consider MSM and MSMW separately in order to expand our knowledge about differential HIV prevention needs for both groups. Such findings can help to develop HIV-prevention interventions that are tailored to the specific needs of these subgroups who remain at high risk of acquiring and transmitting HIV and other STIs. MSMW may benefit from interventions that address the fact that they have sex with both men and women instead of grouping them with MSM in interventions that only focus on sex with men.

Acknowledgments This research was supported in part by grants and a related diversity supplement from the National Institute of Drug Abuse (R01 DA012116-01A2, DA021115-01, and R03 DA021115-02S1) to Thomas L. Patterson.

Open Access This article is distributed under the terms of the Creative Commons Attribution Noncommercial License which permits any noncommercial use, distribution, and reproduction in any medium, provided the original author(s) and source are credited.

\section{References}

Baum, M. K., Rafie, C., Lai, S., Sales, S., Page, B., \& Campa, A. (2009). Crack-cocaine use accelerates HIV disease progression in a cohort of HIV-positive drug users. Journal of Acquired Immune Deficiency Syndromes, 50, 93-99.

Beck, A. T., Steer, R. A., \& Brown, G. K. (1996). Manual for the Beck Depression Inventory-II. San Antonio: Psychological Corporation.

Colfax, G., \& Shoptaw, S. (2005). The methamphetamine epidemic: Implications for HIV prevention and treatment. Current HIV/AIDS Reports, 2, 194-199.

Deiss, R. G., Brouwer, K. C., Loza, O., Lozada, R. M., Ramos, R., Firestone Cruz, M. A., et al. (2008). High-risk sexual and drug using behaviors among male injection drug users who have sex with men in 2 Mexico-US border cities. Sexually Transmitted Diseases, 35, 243-249.

Dodge, B., Jeffries, W. L., \& Sandfort, T. G. M. (2008). Beyond the down low: Sexual risk, protection, and disclosure among at-risk black men who have sex with both men and women (MSMW). Archives of Sexual Behavior, 37, 683-696.

Dodge, B., \& Sandfort, T. G. M. (2007). A review of mental health research on bisexual individuals when compared to homosexual and heterosexual individuals. In B. A. Firestein (Ed.), Becoming visible: Counseling bisexuals across the lifespan (pp. 28-51). New York: Columbia University Press.
Drumright, L. N., Little, S. J., Strathdee, S. A., Slymen, D. J., Araneta, M. R. G., Malcarne, V. L., et al. (2006). Unprotected anal intercourse and substance use among men who have sex with men with recent HIV infection. Journal of Acquired Immune Deficiency Syndrome, 43, 344-350.

Fernández, M. I., Bowen, G. S., Warren, J. C., Ibañez, G. E., Hernandez, N., Harper, G. W., \& Prado, G. (2007). Crystal methamphetamine: A source of added sexual risk for Hispanic men who have sex with men? Drug and Alcohol Dependence, 86, 245-252.

Finlinson, H. A., Colon, H. M., Robles, R. R., \& Soto, M. (2006). Sexual identity formation and AIDS prevention: An exploratory study of non-gay-identified Puerto Rican MSM from working class neighborhoods. AIDS and Behavior, 10, 531-539.

Fisher, J. D., Willcuts, D. L. K., Misovich, S. J., \& Weinstein, B. (1998). Dynamics of sexual risk behavior in HIV-infected men who have sex with men. AIDS and Behavior, 2, 101-113.

Goodenow, C., Netherland, J., \& Szalacha, L. (2002). AIDS-related risk among adolescent males who have sex with males, females, or both: Evidence from a statewide survey. American Journal of Public Health, 92, 203-210.

Gorman, E. M., \& Carroll, R. T. (2000). Substance abuse and HIV: Considerations with regard to methamphetamines and other recreational drugs for nursing practice and research. Journal of the Association of Nurses in AIDS Care, 11, 51-62.

Hart, T. A., Wolitski, R. J., Purcell, D. W., Gomez, C., \& Halkitis, P. (2003). Sexual behavior among HIV-positive men who have sex with men: What's in a label. Journal of Sex Research, 40, 179-188.

Heckman, T. G., Kelly, J. A., Sikkema, K. J., Roffman, R. R., Solomon, L. J., Winett, R. A., et al. (1995). Differences in HIV risk characteristics between bisexual and exclusively gay men. AIDS Education and Prevention, 7, 504-512.

Hightow, L. B., Leone, P. A., MacDonald, D. M., McCoy, S. I., \& Sampson, L. A. (2006). Men who have sex with men and women: A unique risk group for HIV transmission on North Carolina college campuses. Sexually Transmitted Diseases, 33, 585-593.

Jeffries, W. L., \& Dodge, B. (2007). Male bisexuality and condom use at last sexual encounter: Results from a national survey. Journal of Sex Research, 44, 278-289.

Kalichman, S. C., Johnson, J. R., Adair, V., Rompa, D., Multhauf, K., \& Kelly, J. A. (1994). Sexual sensation seeking: Scale development and predicting AIDS-risk behavior among homosexually active men. Journal of Personality Assessment, 62, 385-397.

Kalichman, S. C., \& Rompa, D. (2001). The sexual compulsivity scale: Further development and use with HIV-positive persons. Journal of Personality Assessment, 76, 379-395.

Knight, K. R., Shade, S. B., Purcell, D. W., Rose, C. D., Metsch, L. R., Latka, M. H., et al. (2007). Sexual transmission risk behavior reported among behaviorally bisexual HIV-positive injection drugusing men. Journal of Acquired Immune Deficiency Syndrome, 46, S80-S87.

Lauby, J. L., Millett, G. A., La Pollo, A. B., Bond, L., Murrill, C. S., \& Marks, G. (2008). Sexual risk behaviors of HIV-positive, HIVnegative, and serostatus-unknown black men who have sex with men and women. Archives of Sexual Behavior, 37, 708-719.

Mausbach, B. T., Semple, S. J., Strathdee, S. A., \& Patterson, T. L. (2009). Predictors of safer sex intentions and protected sex among heterosexual HIV-negative methamphetamine users: An expanded model of the theory of planned behavior. AIDS Care, 21, 17-24.

Millet, G., Malebranche, D., Mason, B., \& Spikes, P. (2005). Focusing "down low": Bisexual black men, HIV risk and heterosexual transmission. Journal of the National Medical Association, 97, 52S$59 \mathrm{~S}$.

Montgomery, J. P., Mokotoff, E. D., Gentry, A. C., \& Blair, J. M. (2003). The extent of bisexual behaviour in HIV-infected men and implications for transmission to their female sex partners. AIDS Care, 15, 829-837. 
Muñoz-Laboy, M., \& Dodge, B. (2007). Bisexual Latino men and HIV and sexually transmitted infections risk: An exploratory analysis. American Journal of Public Health, 97, 1102-1106.

Mutchler, M. G., Bogart, L. M., Elliott, M. N., McKay, T., Suttorp, M. J., \& Schuster, M. A. (2008). Psychosocial correlates of unprotected sex without disclosure of HIV-positivity among African-American, Latino, and white men who have sex with men and women. Archives of Sexual Behavior, 37, 736-747.

O'Leary, A. O., Purcell, D. W., Remien, R. H., Fisher, H. E., \& Spikes, P. S. (2007). Characteristics of bisexually active men in the seropositive urban men's study. AIDS Care, 19, 940-946.

Pallonen, U. E., Timpson, S. C., Williams, M. L., \& Ross, M. W. (2009). Stages of consistent condom use, partner intimacy, condom use attitudes, and self-efficacy in African-American crack cocaine users. Archives of Sexual Behavior, 38, 149-158.

Pathela, P., Hajat, A., Schillinger, J., Blank, S., Sell, R., \& Mostashari, F. (2006). Discordance between sexual behavior and self-reported sexual identity: A population-based survey of New York City men. Annals of Internal Medicine, 145, 416-425.

Patterson, T. L., Semple, S. J., Zians, J. K., \& Strathdee, S. A. (2005). Methamphetamine-using HIV-positive men who have sex with men: Correlates of polydrug use. Journal of Urban Health, 82, i121-i126.

Plankey, M. W., Ostrow, D. G., Stall, R., Cox, C., Li, X., Peck, J. A., \& Jacobson, L. P. (2007). The relationship between methamphetamine and popper use and risk of HIV seroconversion in the Multicenter AIDS Cohort Study. Journal of Acquired Immune Deficiency Syndromes, 45, 85-92.
Prabhu, R., Owen, C. L., Folger, K., \& McFarland, W. (2004). The bisexual bridge revisited: Sexual risk behavior among men who have sex with men and women, San Francisco, 1998-2003. AIDS, $18,1604-1606$.

Purcell, D. W., Moss, S., Remien, R. H., Woods, W. J., \& Parsons, J. T. (2005). Illicit substance use, sexual risk, and HIV-positive gay and bisexual men: differences by serostatus of casual partners. AIDS, 19, S37-S47.

Romanelli, F., Smith, K. M., \& Pomeroy, C. (2003). Use of club drugs by $\mathrm{HIV}$-seropositive and HIV-seronegative gay and bisexual men. Topics in HIV Medicine, 11, 25-32.

Sandfort, T. G. M., \& Dodge, B. (2008). “...And then there was the Down Low": Introduction to Black and Latino bisexualities. Archives of Sexual Behavior, 37, 675-682.

Siegel, K., Schrimshaw, E. W., Lekas, H. M., \& Parsons, J. T. (2008). Sexual behaviors of non-gay identified non-disclosing men who have sex with men and women. Archives of Sexual Behavior, 37, 720-735.

Timpson, S. C., Williams, M. L., Bowen, A. M., Atkinson, J. S., \& Ross, M. W. (2010). Sexual activity in HIV-positive African American crack cocaine smokers. Archives of Sexual Behavior, 39, 1353-1358.

Vaudrey, J., Raymond, H. F., Chen, S., Hecht, J., Ahrens, K., \& McFarland, W. (2007). Indicators of use of methamphetamine and other substances among men who have sex with men, San Francisco, 20032006. Drug and Alcohol Dependence, 90, 97-100.

Young, M., Stuber, J., Ahern, J., \& Galea, S. (2005). Interpersonal discrimination and the health of illicit drug users. The American Journal of Drug and Alcohol Abuse, 31, 371-391. 